\title{
POSITIVE DEFINITE FUNCTIONS AND SEBESTYÉN'S OPERATOR MOMENT PROBLEM
}

\author{
ZOLTÁN SEBESTYÉN \\ Department of Applied Analysis, Loránd Eötvös University, 1117 Budapest, \\ Pázmány Péter sétány $1 / C$, Hungary \\ e-mail: sebesty@cs.elte.hu \\ and DAN POPOVICI \\ Department of Mathematics, University of the West, 300223 Timişoara, Bd. V. Pârvan 4, Romania \\ e-mail:popovici@math.uvt.ro
}

(Received 16 November, 2004; accepted 16 May, 2005)

\begin{abstract}
Given a sequence $\left\{A_{n}\right\}_{n \in \mathbb{Z}_{+}}$of bounded linear operators between complex Hilbert spaces $\mathcal{H}$ and $\mathcal{K}$ we characterize the existence of a contraction (resp. isometry, unitary operator, shift) $T$ on $\mathcal{K}$ such that

$$
A_{n}=T^{n} A_{0}, \quad n \in \mathbb{Z}_{+} .
$$

Such moment problems are motivated by their connection with the dilatability of positive operator measures having applications in the theory of stochastic processes.

The solutions, based on the fact that a certain operator function attached to $T$ is positive definite on $\mathbb{Z}$, extend the ones given by Sebestyén in [18], [19] or, recently, by Jabłoński and Stochel in [8]. Some applications, containing new characterizations for isometric, unitary operators, orthogonal projections or commuting pairs having regular dilation, conclude the paper.
\end{abstract}

2000 Mathematics Subject Classification. Primary 47A57, 43A35; Secondary 47A20, 60G10, 47B99.

1. Introduction. One of the most important directions in the theory of nonselfadjoint operators was initiated by the famous theorem of Sz.-Nagy [26] on the existence of a unitary (power) dilation for every contraction operator on a Hilbert space. It has a growing impact on different branches of mathematics and theoretical physics as invariant subspace theory [27], interpolation theory [3] or prediction theory for stationary stochastic processes [9]. Among different proofs for the above mentioned theorem there is one strongly connected to our approach. It uses the fact that every contraction $T$ on a complex Hilbert space is characterized by the property that the function

$$
n \mapsto T(n):= \begin{cases}T^{n}, & n \geq 0 \\ T^{*|n|}, & n<0\end{cases}
$$

is positive definite on $\mathbb{Z}$ (see [27, $\S$ I.8.1]). In other words, by the well-known theorem of Naimark [12], $T(n)$ has an integral representation in terms of a semispectral measure on the unit circle. 
It is our aim in the present paper to give detailed solutions to the following moment problems:

Find necessary and sufficient conditions on a sequence $\left\{h_{n}\right\}_{n \in \mathbb{Z}_{+}}$of vectors in a complex Hilbert space $\mathcal{H}$ to assure the existence of a contraction (isometry, unitary operator, shift) $T$ on $\mathcal{H}$ such that

$$
h_{n}=T^{n} h_{0}, \quad n \geq 0 .
$$

(Problem A; [18])

Replacing $\left\{h_{n}\right\}_{n \in \mathbb{Z}_{+}}$by a sequence $\left\{A_{n}\right\}_{n \in \mathbb{Z}_{+}}$of bounded linear operators between complex Hilbert spaces $\mathcal{H}$ and $\mathcal{K}$ seek a contraction (isometry, unitary operator, shift) $T$ on $\mathcal{K}$, respectively on $\mathcal{H}$ such that

$$
A_{n}=T^{n} A_{0}, \quad n \geq 0,
$$

(Problem B; [19])

respectively

$$
A_{n}=A_{0} T^{n}, \quad n \geq 0 .
$$

(Problem B*).

The discussion above motivates the link between these operator or vector moment problems and the classical moment problem.

While Problem B with contractive or unitary solutions is equivalent to Problem $B^{*}$ by taking adjoints in (4), the solution to Problem A can be obtained by solving Problem B for a particular choice of the sequence $\left\{A_{n}\right\}_{n \in \mathbb{Z}_{+}}$, namely the rank-one operator sequence

$$
A_{n}=h_{n} \otimes h: \mathcal{H} \rightarrow \mathcal{H}, \quad n \geq 0 \quad(h \neq 0 \text { is fixed })
$$

or, alternately,

$$
A_{n}=\mathbf{1}_{\mathbb{C}} h_{n}: \mathbb{C} \rightarrow \mathcal{H}, \quad n \geq 0 .
$$

Such moment problems have been considered before in connection with the theory of stationary or non-stationary stochastic processes, their prediction and filtering.

We should firstly mention that, extending the prediction theory of multivariate stochastic processes developed by Wiener-Masani [28], [29] or Helson-Lowdenslager [6], [7], Suciu and Valuşescu [23], [24] proposed an operator model (a complete correlated action) in which a discrete process is just a sequence $\mathbf{A}=\left\{A_{n}\right\}_{n \in \mathbb{Z}}$ of bounded operators between parameter and measuring spaces of the correlated action. Such a process is stationary if Problem B has a unitary solution (called the shift operator of the process), when $\mathbb{Z}_{+}$is replaced by $\mathbb{Z}$. It is weakly harmonizable if it can be dilated to a stationary process, namely if Problem B has a contractive solution. Moreover, a stationary process $\mathbf{A}=\left\{A_{n}\right\}_{n \in \mathbb{Z}}$ is deterministic, respectively a moving average of a white noise if Problem B for the sequence $\left\{A_{-n}\right\}_{n \in \mathbb{Z}_{+}}$has a unitary, respectively a shift solution on $\mathcal{K}_{0}^{\mathbf{A}}=\bigvee_{n \leq 0} \operatorname{range}\left(A_{n}\right)$.

Similar moment problems have been also used in connection with orthogonally scattered or quasi-isometric measures with applications in the theory of non-stationary stochastic processes [4], [10], [11] (see [8] for a detailed review of these results).

An extensive abstract approach to these problems is due to Sebestyén [18]-[22]. 
Our conditions on the initial data in order to assure a contractive (resp. isometric, resp. shift) solution extend and improve the ones given by Sebestyén [18], [19]. Unitary solutions (see also [8], [22]) are discussed in connection with discrete (deterministic, moving average of a white noise) stationary processes. Some other applications, containing new characterizations for isometric, unitary operators, orthogonal projections or commuting pairs having regular dilation, conclude the paper.

Related multidimensional operator moment problems in connection with multiparameter stochastic processes and the theory of regular dilations will be considered in a future paper (a starting point is given in [8], [17]).

2. Notation and Preliminaries. Let $\mathcal{H}$ and $\mathcal{K}$ be complex Hilbert spaces and $\mathcal{L}(\mathcal{H}, \mathcal{K})(\mathcal{L}(\mathcal{H})$ if $\mathcal{H}=\mathcal{K})$ the Banach space of linear and bounded operators between $\mathcal{H}$ and $\mathcal{K}$. Denote by $\bigvee_{\alpha \in I} \mathcal{H}_{\alpha}$ the closed linear span of a family of subspaces $\left\{\mathcal{H}_{\alpha}\right\}_{\alpha \in I}$ in $\mathcal{H}$.

A function $\Phi: \mathbb{Z} \rightarrow \mathcal{L}(\mathcal{H})$ is positive definite (shortly PD) if $\sum_{n, m \in \mathbb{Z}}\left\langle\Phi(n-m) h_{n}, h_{m}\right\rangle \geq 0$, for every sequence $\left\{h_{n}\right\}_{n \in \mathbb{Z}}$ with finite support. A semispectral measure on the one-dimensional unit circle $\mathbb{T}$ is a function $\sigma \mapsto F(\sigma)$ on the Borel subsets of $\mathbb{T}$ into $\mathcal{L}(\mathcal{H})$ such that, for any $h \in \mathcal{H}$, the map $\sigma \mapsto\langle F(\sigma) h, h\rangle$ is a positive Radon measure on $\mathbb{T}$. There is a bijective correspondence between semispectral measures on $\mathbb{\mathbb { T }}$ into $\mathcal{L}(\mathcal{H})$ and positive definite functions on $\mathbb{Z}$ into $\mathcal{L}(\mathcal{H})$ given by

$$
\Phi(n)=\int_{\mathbb{T}} \bar{\lambda}^{n} d F(\lambda), \quad n \in \mathbb{Z} .
$$

More precisely, by the Naimark dilation theorem [12], [13], the following conditions are equivalent:

(i) $\Phi: \mathbb{Z} \rightarrow \mathcal{L}(\mathcal{H})$ is $\mathrm{PD}$;

(ii) There exists a semispectral measure $F$ on the unit circle into $\mathcal{L}(\mathcal{H})$ such that (5) is satisfied;

(iii) There exist a Hilbert space $\mathcal{K}, V \in \mathcal{L}(\mathcal{H}, \mathcal{K})$ and a unitary operator $U$ on $\mathcal{K}$ such that

$$
\Phi(n)=V^{*} U^{n} V, \quad n \in \mathbb{Z} .
$$

As mentioned earlier, a contraction $T$ on $\mathcal{H}$ can be characterized by the fact that the function $n \mapsto T(n)$ (given by (1)) is PD on $\mathbb{Z}$. By the remark above, there exists a semispectral measure $F$ on the unit circle such that

$$
T^{n}=\int_{\mathbb{T}} \bar{\lambda}^{n} d F(\lambda), \quad n \geq 0 .
$$

Conversely, start with a semispectral measure $F$ on the unit circle into $\mathcal{L}(\mathcal{H})$ and suppose that

$$
\int_{\mathbb{T}} \bar{\lambda}^{n} d F(\lambda)=\left(\int_{\mathbb{T}} \bar{\lambda} d F(\lambda)\right)^{n}, \quad n \geq 0 .
$$

Then there exists a contraction $T$ on $\mathcal{H}$ with (6).

Our problems with contractive solutions can be then reformulated as:

Find necessary and sufficient conditions on $\left\{h_{n}\right\}_{n \in \mathbb{Z}_{+}} \subset \mathcal{H}$, respectively on $\left\{A_{n}\right\}_{n \in \mathbb{Z}_{+}} \subset$ $\mathcal{L}(\mathcal{H}, \mathcal{K})$ to assure the existence of an operator valued semispectral measure $F$ on the unit 
circle which satisfies (7) and such that:

$$
h_{n}=\int_{\mathbb{T}} \bar{\lambda}^{n} d F(\lambda) h_{0}, \quad n \geq 0,
$$

(Problem A)

respectively

$$
A_{n} h=\int_{\mathbb{T}} \bar{\lambda}^{n} d F(\lambda) A_{0} h, \quad h \in \mathcal{H}, n \geq 0,
$$

(Problem B)

or

$$
A_{n} h=\int_{\mathbb{T}} \bar{\lambda}^{n} d A_{0} F(\lambda) h, \quad h \in \mathcal{H}, n \geq 0 .
$$

(Problem B*)

A (unilateral) shift is an operator $S$ on a Hilbert space $\mathcal{H}$ unitarily equivalent with the multiplication by the independent variable on a certain Hardy space on the unit circle. By the Wold decomposition theorem [5] an isometric operator $S$ on $\mathcal{H}$ is a shift if and only if it has no unitary part, namely if $\bigcap_{n \geq 0} S^{n} \mathcal{H}=\{0\}$.

As already mentioned in the introduction, extending the Wiener-Masani approach to the prediction theory for finite multivariate stationary processes, Suciu and Valuşescu [23], [24] proposed a mathematical model for the infinite multivariate case. They considered the action of operators in $\mathcal{L}(\mathcal{H})(\mathcal{H}$ being a Hilbert space as the parameter space) on a right $\mathcal{L}(\mathcal{H})$-module $H$ (called the state space). The couple $\{\mathcal{H}, H\}$ is said to be a correlated action of $\mathcal{L}(\mathcal{H})$ on $H$ if there exists an "inner product" $\Gamma: H \times H \rightarrow \mathcal{L}(\mathcal{H})$. Usually $H=\mathcal{L}(\mathcal{H}, \mathcal{K})(\mathcal{K}$ is a Hilbert space as the measuring space $), \Gamma(S, T):=S^{*} T(S, T \in H)$ and the correlated action is, in this case, complete. A discrete stationary process is just a sequence $\mathbf{A}=\left\{A_{n}\right\}_{n \in \mathbb{Z}} \subset \mathcal{L}(\mathcal{H}, \mathcal{K})$ such that there exists a unitary operator $U$ on $\mathcal{K}$ with $A_{n}=U^{n} A_{0}, n \in \mathbb{Z}$. A stationary process $\mathbf{A}$ is a white noise if it has pairwise orthogonal ranges. The restriction $U_{\mathbf{A}}$ of $U$ to the measuring space $\mathcal{K}^{\mathbf{A}}=\bigvee_{n \in \mathbb{Z}}$ range $\left(A_{n}\right)$ of $\mathbf{A}$ is a unitary operator uniquely determined by $\mathbf{A}$ and called the shift operator of $\mathbf{A}$. By Wold's theorem ([23]), every stationary process $\mathbf{A}$ can be uniquely decomposed into the orthogonal sum between a deterministic process (it coincides with its predictable part) and a moving average of a white noise (the part "corrupted" by noises). As the past and present measuring space $\mathcal{K}_{0}^{\mathbf{A}}=\bigvee_{n \leq 0} \operatorname{range}\left(A_{n}\right)$ is invariant to $U_{\mathbf{A}}^{*}$, we can consider the isometric operator $V_{\mathbf{A}}=\left.U_{\mathbf{A}}^{*}\right|_{\mathcal{K}_{0}^{\mathbf{A}}}$. It can be used to characterize special classes of stationary processes $\mathbf{A}: \mathbf{A}$ is deterministic iff $V_{\mathbf{A}}$ is unitary; $\mathbf{A}$ is a moving average of a white noise iff $V_{\mathbf{A}}$ is shift.

As proved by Sz.-Nagy [26], any given operator $T$ on $\mathcal{H}$ is a contraction if and only if it has a unitary (power) dilation: a unitary operator $U$ on a Hilbert space $\mathcal{K}$ (containing $\mathcal{H}$ as a closed subspace) such that

$$
T^{n} h=P_{\mathcal{H}} U^{n} h, \quad h \in \mathcal{H}, n \in \mathbb{Z}_{+} .
$$

A discrete process $\mathbf{A}=\left\{A_{n}\right\}_{n \in \mathbb{Z}}$ on $\mathcal{H}$ is weakly harmonizable if it can be dilated to a stationary process $\mathbf{B}=\left\{B_{n}\right\}_{n \in \mathbb{Z}}$ on $\mathcal{K}$ such that $A_{n}=\left.P_{\mathcal{H}}^{\mathcal{K}} B_{n}\right|_{\mathcal{H}}, n \in \mathbb{Z}$; in particular, if $T(\cdot)$ is given by $(1)$ and $A_{0} \in \mathcal{L}(\mathcal{H})$ then the process $\left\{T(\cdot) A_{0}\right\}_{n \in \mathbb{Z}}$ is weakly harmonizable; this fact motivates our study of Problem B with contractive solutions. 
Ando [1] extended Sz.-Nagy's result to a pair $\mathbf{T}=\left(T_{1}, T_{2}\right)$ of commuting contractions on $\mathcal{H}$ : there exists a pair $\mathbf{U}=\left(U_{1}, U_{2}\right)$ of commuting unitary operators on a Hilbert space $\mathcal{K}$ (containing $\mathcal{H}$ as a closed subspace) such that

$$
T_{1}^{n_{1}} T_{2}^{n_{2}} h=P_{\mathcal{H}} U_{1}^{n_{1}} U_{2}^{n_{2}} h, \quad h \in \mathcal{H}, n_{1}, n_{2} \in \mathbb{Z}_{+} .
$$

A unitary dilation $\mathbf{U}=\left(U_{1}, U_{2}\right)$ of $\mathbf{T}=\left(T_{1}, T_{2}\right)$ is said to be regular ([2], [25]) if, moreover,

$$
T_{1}^{* n_{1}} T_{2}^{n_{2}} h=P_{\mathcal{H}} U_{1}^{* n_{1}} U_{2}^{n_{2}} h, \quad h \in \mathcal{H}, n_{1}, n_{2} \in \mathbb{Z}_{+} .
$$

Regular dilations have been introduced and extensively studied by Brehmer ([2]; see also [25]). The following equivalent conditions are also due to him:

(i) $\mathbf{T}$ has a regular dilation;

(ii) $\Delta_{\mathrm{T}}=I-T_{1}^{*} T_{1}-T_{2}^{*} T_{2}+T_{1}^{*} T_{2}^{*} T_{1} T_{2} \geq 0$;

(iii) The function

$$
\mathbb{Z}^{2} \ni\left(n_{1}, n_{2}\right) \mapsto \begin{cases}T_{1}^{n_{1}} T_{2}^{n_{2}}, & n_{1}, n_{2} \geq 0 \\ T_{2}^{*\left|n_{2}\right|} T_{1}^{n_{1}}, & n_{1} \geq 0, n_{2}<0 \\ T_{1}^{*\left|n_{1}\right|} T_{2}^{n_{2}}, & n_{1}<0, n_{2} \geq 0 \\ T_{1}^{*\left|n_{1}\right|} T_{2}^{*\left|n_{2}\right|}, & n_{1}, n_{2}<0\end{cases}
$$

is positive definite on $\mathbb{Z}^{2}$.

Regular dilations have been recently used in connection to various kind of applications in [14]-[17].

\section{Solutions.}

3.1. Contractive Solutions. The following solution to Problem $B$ extends the one given by Sebestyén in [19].

THEOREM 3.1. Let $\left\{A_{n}\right\}_{n \geq 0} \subset \mathcal{L}(\mathcal{H}, \mathcal{K})$. The following conditions are equivalent:

(i) Problem $B$ has a contractive solution;

(ii)

$$
\left\|\sum_{m, m^{\prime}} A_{m+m^{\prime}}\left(h_{m, m^{\prime}}\right)\right\|^{2} \leq \sum_{m, m^{\prime}, n, n^{\prime}}\left\langle A_{(m-n)^{+}+m^{\prime}}\left(h_{m, m^{\prime}}\right), A_{(m-n)^{-}+n^{\prime}}\left(h_{n, n^{\prime}}\right)\right\rangle,
$$

(iii)

for every finite double sequence $\left\{h_{n, n^{\prime}}\right\}_{n, n^{\prime} \geq 0}$ of vectors in $\mathcal{H}$;

$$
\sum_{m, m^{\prime}, n, n^{\prime}}\left\langle A_{(m-n)^{+}+m^{\prime}}\left(h_{m, m^{\prime}}\right), A_{(m-n)^{-}+n^{\prime}}\left(h_{n, n^{\prime}}\right)\right\rangle \geq 0
$$

for every finite double sequence $\left\{h_{n, n^{\prime}}\right\}_{n, n^{\prime} \geq 0}$ of vectors in $\mathcal{H}$; 
(iv)

$$
\left\|\sum_{n \geq 0} A_{n+1}\left(h_{n}\right)\right\| \leq\left\|\sum_{n \geq 0} A_{n}\left(h_{n}\right)\right\|,
$$

for every finite sequence $\left\{h_{n}\right\}_{n \geq 0}$ of vectors in $\mathcal{H}$

(for $n \in \mathbb{Z}$ notations $n^{+}=\max \{n, 0\}$ and $n^{-}=-\min \{n, 0\}$ are used ).

Proof. (i) $\Rightarrow$ (ii) Suppose that there exists a contraction $T$ on $\mathcal{K}$ such that

$$
A_{n}=T^{n} A_{0}, \quad n \geq 0 .
$$

If $U$ is a unitary dilation of $T$ then, by (8),

$$
\begin{aligned}
& \left\|\sum_{m, m^{\prime}} A_{m+m^{\prime}}\left(h_{m, m^{\prime}}\right)\right\|^{2} \\
& =\left\|\sum_{m, m^{\prime}} T^{m} A_{m^{\prime}}\left(h_{m, m^{\prime}}\right)\right\|^{2} \\
& \leq\left\|\sum_{m, m^{\prime}} U^{m} A_{m^{\prime}}\left(h_{m, m^{\prime}}\right)\right\|^{2} \\
& =\sum_{m, m^{\prime}, n, n^{\prime}}\left\langle U^{m} A_{m^{\prime}}\left(h_{m, m^{\prime}}\right), U^{n} A_{n^{\prime}}\left(h_{n, n^{\prime}}\right)\right\rangle \\
& =\sum_{m, m^{\prime}, n, n^{\prime}}\left\langle T^{(m-n)^{+}} A_{m^{\prime}}\left(h_{m, m^{\prime}}\right), T^{(m-n)^{-}} A_{n^{\prime}}\left(h_{n, n^{\prime}}\right)\right\rangle \\
& =\sum_{m, m^{\prime}, n, n^{\prime}}\left\langle A_{(m-n)^{+}+m^{\prime}}\left(h_{m, m^{\prime}}\right), A_{(m-n)^{-}+n^{\prime}}\left(h_{n, n^{\prime}}\right)\right\rangle,
\end{aligned}
$$

for every finite sequence $\left\{h_{m, m^{\prime}}\right\}_{m, m^{\prime}} \subset \mathcal{H}$.

(ii) $\Rightarrow$ (iii) is obvious.

(iii) $\Rightarrow$ (iv) The following computations

$$
\begin{aligned}
\sum_{n \geq 0}\left\|\sum_{\substack{m \geq n \\
m^{\prime} \geq 0}} A_{m-n+m^{\prime}}\left(h_{m, m^{\prime}}\right)\right\|^{2} \\
=\sum_{n \geq 0}\left\langle\sum_{\substack{p \geq n \\
p^{\prime} \geq 0}} A_{p-n+p^{\prime}}\left(h_{p, p^{\prime}}\right), \sum_{\substack{q \geq n \\
q^{\prime} \geq 0}} A_{q-n+q^{\prime}}\left(h_{q, q^{\prime}}\right)\right\rangle \\
\left.=\sum_{n \geq 0}\left\langle\sum_{\substack{p \geq n \\
p^{\prime} \geq 0}} A_{p-n+p^{\prime}}\left(h_{p, p^{\prime}}\right), \sum_{\substack{q^{\prime} \geq 0 \\
q_{q^{\prime}}}} A_{n, q^{\prime}}\right)\right\rangle \\
\quad+\sum_{n \geq 0}\left\langle\sum_{p^{\prime} \geq 0} A_{p^{\prime}}\left(h_{n, p^{\prime}}\right), \sum_{\substack{q>n \\
q^{\prime} \geq 0}} A_{q-n+q^{\prime}}\left(h_{q, q^{\prime}}\right)\right\rangle+\sum_{n \geq 0}\left\|\sum_{\substack{m>n \\
m^{\prime} \geq 0}} A_{m-n+m^{\prime}}\left(h_{m, m^{\prime}}\right)\right\|^{2}
\end{aligned}
$$




$$
=\sum_{m, m^{\prime}, n, n^{\prime}}\left\langle A_{(m-n)^{+}+m^{\prime}}\left(h_{m, m^{\prime}}\right), A_{(m-n)^{-}+n^{\prime}}\left(h_{n, n^{\prime}}\right)\right\rangle+\sum_{k \geq 1}\left\|\sum_{\substack{m \geq k \\ m \geq 0}} A_{m-k+m^{\prime}+1}\left(h_{m, m^{\prime}}\right)\right\|^{2}
$$

show that, by (iii),

$$
\sum_{n \geq 1}\left\|\sum_{\substack{m \geq n \\ m^{\prime} \geq 0}} A_{m-n+m^{\prime}+1}\left(h_{m, m^{\prime}}\right)\right\|^{2} \leq \sum_{n \geq 0}\left\|\sum_{\substack{m \geq n \\ m^{\prime} \geq 0}} A_{m-n+m^{\prime}}\left(h_{m, m^{\prime}}\right)\right\|^{2}
$$

for every finite double sequence $\left\{h_{n, n^{\prime}}\right\}_{n, n^{\prime} \geq 0}$ of vectors in $\mathcal{H}$.

Let $\left\{h_{n}\right\}_{n \geq 0}$ be any finite sequence of vectors in $\mathcal{H}$. We compute (9) with

$$
\begin{aligned}
h_{m, m^{\prime}} & =0, \quad \text { for }(m>1) \text { or }\left(m=m^{\prime}=0\right), \\
h_{0, m^{\prime}} & =-h_{m^{\prime}-1} \quad\left(m^{\prime} \geq 1\right) \quad \text { and } \\
h_{1, m^{\prime}} & =h_{m^{\prime}} \quad\left(m^{\prime} \geq 0\right)
\end{aligned}
$$

to finally obtain (iv).

(iv) $\Rightarrow(i)$ Let $\mathcal{K}_{0}$ be the closed linear subspace of $\mathcal{K}$ generated by all vectors of the form $A_{n}(h), n \geq 0, h \in \mathcal{H}$.

We define $T$ on $\mathcal{K}_{0}$ as

$$
\mathcal{K}_{0} \ni \sum_{n \geq 0} A_{n}\left(h_{n}\right) \mapsto \sum_{n \geq 0} A_{n+1}\left(h_{n}\right) \in \mathcal{K}_{0}
$$

which, by (iv), is a well-defined contraction. Obviously $T A_{n}(h)=A_{n+1}(h)(n \geq 0, h \in$ $\mathcal{H}$ ) and $T$ can be extended to a contraction on the whole $\mathcal{K}$.

We obtain, as a consequence, a solution to Problem $A$, which extends the one in [18]. For the multidimensional case we refer to [17].

COROLlary 3.2. Let $\left\{h_{n}\right\}_{n \geq 0} \subset \mathcal{H}$. The following conditions are equivalent:

(i) Problem A has a contractive solution;

(ii)

$$
\left\|\sum_{m, m^{\prime}} c_{m, m^{\prime}} h_{m+m^{\prime}}\right\|^{2} \leq \sum_{m, m^{\prime}, n, n^{\prime}} c_{m, m^{\prime}} \overline{c_{n, n^{\prime}}}\left\langle h_{(m-n)^{+}+m^{\prime}}, h_{(m-n)^{-}+n^{\prime}}\right\rangle,
$$

for every finite double sequence $\left\{c_{n, n^{\prime}}\right\}_{n, n^{\prime} \geq 0}$ of complex numbers;

(iii)

$$
\sum_{m, m^{\prime}, n, n^{\prime}} c_{m, m^{\prime}} \overline{c_{n, n^{\prime}}}\left(h_{(m-n)^{+}+m^{\prime}}, h_{(m-n)^{-}+n^{\prime}}\right) \geq 0,
$$

(iv)

for every finite double sequence $\left\{c_{n, n^{\prime}}\right\}_{n, n^{\prime} \geq 0}$ of complex numbers;

$$
\left\|\sum_{n \geq 0} c_{n} h_{n+1}\right\| \leq\left\|\sum_{n \geq 0} c_{n} h_{n}\right\|
$$

for every finite sequence $\left\{c_{n}\right\}_{n \geq 0}$ of complex numbers. 
REMARK 3.3. Denote by $T_{0}$ the contraction on $\mathcal{K}_{0}=\bigvee_{n \geq 0} h_{n}$ (resp. $\mathcal{K}_{0}=$ $\left.\bigvee_{n \geq 0} \operatorname{range}\left(A_{n}\right)\right)$ defined by

$$
\mathcal{K}_{0} \ni \sum_{n} c_{n} h_{n} \mapsto \sum_{n} c_{n} h_{n+1} \in \mathcal{K}_{0}
$$

(resp. by (10)). Any contractive solution of Problem $A$ (resp. Problem $B$ ) has a matrix representation of the form

$$
T=\left(\begin{array}{cc}
T_{0} & A \\
0 & B
\end{array}\right)
$$

relative to the orthogonal decomposition $\left.\mathcal{K}=\mathcal{K}_{0} \oplus\left(\mathcal{K} \ominus \mathcal{K}_{0}\right), A \in \mathcal{L}\left(\mathcal{K} \ominus \mathcal{K}_{0}, \mathcal{K}_{0}\right)\right)$ and $B \in \mathcal{L}\left(\mathcal{K} \ominus \mathcal{K}_{0}\right)$ being arbitrary such that

$$
\left\|T_{0} k_{0}+A k_{0}^{\prime}\right\|^{2}+\left\|B k_{0}^{\prime}\right\|^{2} \leq\left\|k_{0}\right\|^{2}+\left\|k_{0}^{\prime}\right\|^{2}, \quad k_{0} \in \mathcal{K}_{0}, k_{0}^{\prime} \in \mathcal{K} \ominus \mathcal{K}_{0} .
$$

Consequently, the solution to our problem is unique if and only if

$$
\mathcal{K}=\mathcal{K}_{0}
$$

ReMARK 3.4. Let $T \in \mathcal{L}(\mathcal{H})$. Apply Theorem 3.1 (ii) to the sequence $A_{n}=T^{n}(n \geq$ 0 ) to deduce that $T$ is contractive if and only if

$$
\left\|\sum_{m} T^{m} h_{m}\right\|^{2} \leq \sum_{m, n}\left\langle T^{(m-n)^{+}} h_{m}, T^{(m-n)^{-}} h_{n}\right\rangle
$$

for every finite sequence $\left\{h_{n}\right\}_{n \geq 0}$ of vectors in $\mathcal{H}$.

3.2. Isometric Solutions. Problem $B$ for a sequence $\left\{A_{n}\right\}_{n \geq 0} \subset \mathcal{L}(\mathcal{H}, \mathcal{K})$ has an isometric solution if and only if (10) defines an isometric operator on $\mathcal{K}_{0}$, i.e.

$$
\left\|\sum_{n \geq 0} A_{n+1}\left(h_{n}\right)\right\|=\left\|\sum_{n \geq 0} A_{n}\left(h_{n}\right)\right\|,
$$

for every finite sequence $\left\{h_{n}\right\}_{n \geq 0}$ of vectors in $\mathcal{H}$. Equivalently,

$$
A_{m+1}^{*} A_{n+1}=A_{m}^{*} A_{n}, \quad m, n \geq 0 .
$$

The following characterization shows that a contractive solution can be chosen to be isometric if equality holds in Theorem 3.1 (ii).

Proposition 3.5. Let $\left\{A_{n}\right\}_{n \geq 0} \subset \mathcal{L}(\mathcal{H}, \mathcal{K})$. Then Problem $B$ has an isometric solution if and only if

$$
\left\|\sum_{m, m^{\prime}} A_{m+m^{\prime}}\left(h_{m, m^{\prime}}\right)\right\|^{2}=\sum_{m, m^{\prime}, n, n^{\prime}}\left\langle A_{(m-n)^{+}+m^{\prime}}\left(h_{m, m^{\prime}}\right), A_{(m-n)^{-}+n^{\prime}}\left(h_{n, n^{\prime}}\right)\right\rangle,
$$

for every finite double sequence $\left\{h_{n, n^{\prime}}\right\}_{n, n^{\prime} \geq 0}$ of vectorsin $\mathcal{H}$. 
Proof. The proof follows by an argument similar to the one of Theorem A in [19]. Necessity: Suppose that Problem $B$ has an isometric solution $V$. Then

$$
\begin{aligned}
\left\|\sum_{m, m^{\prime}} A_{m+m^{\prime}}\left(h_{m, m^{\prime}}\right)\right\|^{2} \\
=\left\|\sum_{m, m^{\prime}} V^{m} A_{m^{\prime}}\left(h_{m, m^{\prime}}\right)\right\|^{2} \\
=\sum_{m, m^{\prime}, n, n^{\prime}}\left\langle V^{m} A_{m^{\prime}}\left(h_{m, m^{\prime}}\right), V^{n} A_{n^{\prime}}\left(h_{n, n^{\prime}}\right)\right\rangle \\
=\sum_{m, m^{\prime}, n, n^{\prime}}\left\langle A_{(m-n)^{+}+m^{\prime}}\left(h_{m, m^{\prime}}\right), A_{(m-n)^{-}+n^{\prime}}\left(h_{n, n^{\prime}}\right)\right\rangle,
\end{aligned}
$$

for every finite sequence $\left\{h_{m, m^{\prime}}\right\}_{m, m^{\prime}} \subset \mathcal{H}$.

Sufficiency: Let $\mathcal{K}_{0}=\bigvee_{n \geq 0} \operatorname{range}\left(A_{n}\right)$.

If (16) holds, let $\mathcal{F}_{0}$ be the linear space of all finite double sequences $\left\{h_{n, n^{\prime}}\right\}_{n, n^{\prime} \geq 0}$ of vectors in $\mathcal{H}$ endowed with the semi-inner product

$$
\left\langle\left\{h_{m, m^{\prime}}\right\},\left\{h_{m, m^{\prime}}^{\prime}\right\}\right\rangle:=\sum_{m, m^{\prime}, n, n^{\prime}}\left\langle A_{(m-n)^{+}+m^{\prime}}\left(h_{m, m^{\prime}}\right), A_{(m-n)^{-}+n^{\prime}}\left(h_{n, n^{\prime}}^{\prime}\right)\right\rangle .
$$

By factoring and completing $\mathcal{F}_{0}$ becomes a Hilbert space $\mathcal{F}$. The map

$$
\mathcal{F} \ni\left\{h_{m, m^{\prime}}\right\} \stackrel{U}{\mapsto} \sum_{m, m^{\prime}} A_{m+m^{\prime}}\left(h_{m, m^{\prime}}\right) \in \mathcal{K}_{0}
$$

is clearly a unitary operator, while the shift on $\mathcal{F}$,

$$
S\left\{h_{m, m^{\prime}}\right\}=\left\{h_{m, m^{\prime}}^{\prime}\right\}, \text { where } h_{m, m^{\prime}}^{\prime}=h_{m-1, m^{\prime}}(m \geq 1) \text { and } h_{0, m^{\prime}}^{\prime}=0
$$

is isometric. Then $V:=U S U^{*}$ is isometric on $\mathcal{K}_{0}$, can be extended isometrically to the whole $\mathcal{K}$ and $V A_{k}=A_{k+1}(k \geq 0)$.

COROLlary 3.6. Let $\left\{h_{n}\right\}_{n \geq 0} \subset \mathcal{H}$. Then Problem A has an isometric solution if and only if

$$
\left\|\sum_{m, m^{\prime}} c_{m, m^{\prime}} h_{m+m^{\prime}}\right\|^{2}=\sum_{m, m^{\prime}, n, n^{\prime}} c_{m, m^{\prime}} \overline{c_{n, n^{\prime}}}\left\langle h_{(m-n)^{+}+m^{\prime}}, h_{(m-n)^{-}+n^{\prime}}\right\rangle,
$$

for every finite double sequence $\left\{c_{n, n^{\prime}}\right\}_{n, n^{\prime} \geq 0}$ of complex numbers.

REMARK 3.7. Let $V_{0}$ be the isometric operator on $\mathcal{K}_{0}$ defined by (11) (resp. (10)). Observe that any isometric solution to our problem has the matrix representation

$$
V=\left(\begin{array}{cc}
V_{0} & A \\
0 & B
\end{array}\right)
$$

relative to the decomposition $\mathcal{K}=\mathcal{K}_{0} \oplus\left(\mathcal{K} \ominus \mathcal{K}_{0}\right), \quad A \in \mathcal{L}\left(\mathcal{K} \ominus \mathcal{K}_{0}, \mathcal{K}_{0}\right)$ and $B \in$ $\mathcal{L}\left(\mathcal{K} \ominus \mathcal{K}_{0}\right)$ being arbitrary such that $A^{*} A+B^{*} B=I_{\mathcal{K} \ominus \mathcal{K}_{0}}$ and $\operatorname{range}(A) \perp \mathcal{K}_{1}=$ $\bigvee_{n \geq 1} \operatorname{range}\left(A_{n}\right)\left(\right.$ resp. $\left.\operatorname{range}(A) \perp \mathcal{K}_{1}=\bigvee_{n \geq 1} h_{n}\right)$. 
Deduce that an isometric solution is unique if and only if (12) is satisfied.

REMARK 3.8. An operator $V \in \mathcal{L}(\mathcal{H})$ is isometric if and only if equality holds in (13):

$$
\left\|\sum_{m} V^{m}\left(h_{m}\right)\right\|^{2}=\sum_{m, n}\left\langle V^{(m-n)^{+}}\left(h_{m}\right), V^{(m-n)^{-}}\left(h_{n}\right)\right\rangle,
$$

for every finite sequence $\left\{h_{n}\right\}_{n \geq 0}$ of vectors in $\mathcal{H}$.

3.3. Unitary Solutions. Start with a bilateral sequence $\mathbf{A}=\left\{A_{n}\right\}_{n \in \mathbb{Z}} \subset \mathcal{L}(\mathcal{H}, \mathcal{K})$. Recall that $\mathbf{A}$ is a discrete stationary process if there exists a unitary operator $U$ on $\mathcal{K}$ with

$$
A_{n}=U^{n} A_{0}, \quad n \in \mathbb{Z}
$$

It is well-known (see, for example, [23]) that $\mathbf{A}$ is a discrete stationary process if and only if

$$
A_{m+1}^{*} A_{n+1}=A_{m}^{*} A_{n}, \quad m, n \in \mathbb{Z} .
$$

If $\mathbf{A}$ satisfies the positivity condition

$$
\sum_{m, n, p, q \in \mathbb{Z}}\left\langle A_{m+q} h_{m, n}, A_{n+p} h_{p, q}\right\rangle \geq 0
$$

for every finite double sequence $\left\{h_{m, n}\right\}_{m, n \in \mathbb{Z}} \subset \mathcal{H}$, it is proved in [8] that (19) can be replaced by

$$
A_{1}^{*} A_{1}=A_{0}^{*} A_{0} \text { and } A_{n+1}^{*} A_{0}=A_{n}^{*} A_{-1}, \quad n \in \mathbb{Z} .
$$

If (18) has a unitary solution $U$ then obviously the function

$$
\mathbb{Z} \ni n \mapsto A_{0}^{*} A_{n} \in \mathcal{L}(\mathcal{H})
$$

is $\mathrm{PD}$ on $\mathbb{Z}$. The converse is not true even if we further suppose that

$$
A_{n}^{*} A_{n}=A_{0}^{*} A_{0}, \quad n \in \mathbb{Z} .
$$

EXAMPLE 3.9. Let $Z$ be a unitary operator on $\mathcal{H}, \mathcal{H}_{0}$ an infinite dimensional subspace invariant (but not double invariant) for $Z$ and $P=I-P_{\mathcal{H}_{0}}$. Consider the sequence

$$
A_{n}=Z^{n} P-2 k_{n} \otimes\left(P Z^{-n} k_{n}\right), \quad n \in \mathbb{Z},
$$

$\left\{k_{n}\right\}_{n \in \mathbb{Z}} \subset \mathcal{H}_{0}$ being an orthonormal sequence with $k_{1} \perp Z \mathcal{H}_{0}$ and $k_{2} \in Z \mathcal{H}_{0}$. Easy computations show that

$$
\begin{aligned}
A_{m}^{*} A_{n}= & P Z^{n-m} P-2\left(P Z^{-m} k_{n}\right) \otimes\left(P Z^{-n} k_{n}\right)-2\left(P Z^{-m} k_{m}\right) \otimes\left(P Z^{-n} k_{m}\right) \\
& +4\left\langle k_{n}, k_{m}\right\rangle\left(P Z^{-m} k_{m}\right) \otimes\left(P Z^{-n} k_{n}\right), \quad m, n \in \mathbb{Z} .
\end{aligned}
$$


Then $A_{n}^{*} A_{n}=P, n \in \mathbb{Z}$ and

$$
\sum_{m, n}\left\langle A_{m-n}\left(h_{m}\right), A_{0}\left(h_{n}\right)\right\rangle=\sum_{m, n}\left\langle P Z^{m-n} P h_{m}, h_{n}\right\rangle=\left\|\sum_{n} Z^{n} P h_{n}\right\|^{2} \geq 0,
$$

for every finite sequence $\left\{h_{n}\right\}_{n \in \mathbb{Z}} \subset \mathcal{H}$.

Moreover,

$$
A_{2}^{*} A_{1}=P Z^{*} P-2\left(Z^{-2} k_{1}\right) \otimes\left(Z^{-1} k_{1}\right) \neq P Z^{*} P=A_{1}^{*} A_{0}
$$

and our problem cannot have a unitary solution.

The following characterization clarifies the connection between our problem and positive definiteness of (22).

Proposition 3.10. A is a discrete stationary process if and only if

$$
\left\|\sum_{m} A_{m}\left(h_{m}\right)\right\|^{2}=\sum_{m, n}\left\langle A_{m-n}\left(h_{m}\right), A_{0}\left(h_{n}\right)\right\rangle
$$

for every finite sequence $\left\{h_{n}\right\}_{n \in \mathbb{Z}}$ of vectors in $\mathcal{H}$.

Proof. We proceed as in the proof of Proposition 3.5.

Necessity: If (18) holds then

$$
\begin{aligned}
\left\|\sum_{m} A_{m}\left(h_{m}\right)\right\|^{2} & =\sum_{m, n}\left\langle A_{m}\left(h_{m}\right), A_{n}\left(h_{n}\right)\right\rangle \\
& =\sum_{m, n}\left\langle U^{m-n} A_{0}\left(h_{m}\right), A_{0}\left(h_{n}\right)\right\rangle \\
& =\sum_{m, n}\left\langle A_{m-n}\left(h_{m}\right), A_{0}\left(h_{n}\right)\right\rangle,
\end{aligned}
$$

for every finite sequence $\left\{h_{n}\right\}_{n \in \mathbb{Z}}$ of vectors in $\mathcal{H}$.

Sufficiency: Let $\mathcal{K}^{\mathbf{A}}=\bigvee_{n \in \mathbb{Z}} \operatorname{range}\left(A_{n}\right)$.

If (24) holds, let $\mathcal{F}_{0}$ be the linear space of all finite sequences $\left\{h_{n}\right\}_{n \in \mathbb{Z}}$ of vectors in $\mathcal{H}$ endowed with the semi-inner product

$$
\left\langle\left\{h_{m}\right\}_{m},\left\{h_{m}^{\prime}\right\}_{m}\right\rangle:=\sum_{m, n}\left\langle A_{m-n}\left(h_{m}\right), A_{0}\left(h_{n}^{\prime}\right)\right\rangle .
$$

The map

$$
\mathcal{F}_{0} \ni\left\{h_{m}\right\}_{m \in \mathbb{Z}} \mapsto \sum_{m} A_{m}\left(h_{m}\right) \in \mathcal{K}^{\mathbf{A}}
$$

can be extended to a unitary operator $W$ on the Hilbert space $\mathcal{F}$ (obtained by factoring and completing $\mathcal{F}_{0}$ ). If $B$ is the bilateral shift on $\mathcal{F}$ then $U=W B W^{*}$ is unitary on $\mathcal{K}^{\mathbf{A}}$ and $U A_{n}=A_{n+1}, n \in \mathbb{Z}$.

$U$ can be extended to a unitary operator on $\mathcal{K}$. 
REMARK 3.11. If $\mathbf{A}=\left\{A_{n}\right\}_{n \in \mathbb{Z}}$ has pairwise orthogonal ranges then condition (23) is sufficient (and also necessary) to define a stationary process (in this case $\mathbf{A}$ is a white noise).

Corollary 3.12. Let $\mathbf{h}=\left\{h_{n}\right\}_{n \in \mathbb{Z}} \subset \mathcal{H}$. The following conditions are equivalent:

(i) There exists a unitary operator $U$ on $\mathcal{H}$ such that

$$
h_{n}=U^{n} h_{0}, \quad n \in \mathbb{Z}
$$

(ii)

$$
\left\|\sum_{m} c_{m} h_{m}\right\|^{2}=\sum_{m, n} c_{m} \overline{c_{n}}\left\langle h_{m-n}, h_{0}\right\rangle
$$

for every finite sequence $\left\{c_{n}\right\}_{n \in \mathbb{Z}}$ of complex numbers.

REMARK 3.13. Any unitary solution of equation (25), resp. (18) has the form

$$
U=U_{0} \oplus U_{1}
$$

with $U_{0}$ defined by

$$
\mathcal{K}^{\mathbf{h}}:=\bigvee_{n \in \mathbb{Z}} h_{n} \ni \sum_{n \in \mathbb{Z}} c_{n} h_{n} \mapsto \sum_{n \in \mathbb{Z}} c_{n} h_{n+1} \in \mathcal{K}^{\mathbf{h}},
$$

resp. by

$$
\mathcal{K}^{\mathbf{A}} \ni \sum_{n \in \mathbb{Z}} A_{n}\left(h_{n}\right) \mapsto \sum_{n \in \mathbb{Z}} A_{n+1}\left(h_{n}\right) \in \mathcal{K}^{\mathbf{A}}
$$

and $U_{1}$ an arbitrary unitary operator on $\mathcal{K} \ominus \mathcal{K}^{\mathbf{h}}$, resp. $\mathcal{K} \ominus \mathcal{K}^{\mathbf{A}}$.

Such a solution is unique if and only if $\mathcal{K}=\mathcal{K}^{\mathbf{h}}$, resp. $\mathcal{K}=\mathcal{K}^{\mathbf{A}}$.

REMARK 3.14. Take $A_{n}=U^{n}(n \geq 0), A_{n}=U^{*|n|}(n<0)$ for an operator $U \in$ $\mathcal{L}(\mathcal{H})$ and apply Proposition 3.10. Obtain that $U$ is unitary if and only if

$$
\left\|\sum_{n \geq 0} U^{n} h_{n}+\sum_{n \geq 1} U^{* n} h_{-n}\right\|^{2}=\sum_{m, n}\left\langle U^{(m-n)^{+}} h_{m}, U^{(m-n)^{-}} h_{n}\right\rangle,
$$

for every finite sequence $\left\{h_{n}\right\}_{n \in \mathbb{Z}}$ of vectors in $\mathcal{H}$.

Let $\mathbf{A}=\left\{A_{n}\right\}_{n \in \mathbb{Z}} \subset \mathcal{L}(\mathcal{H}, \mathcal{K})$ be a discrete stationary process. Denote by $U_{\mathbf{A}}$ the shift operator of the process (defined on $\mathcal{K}^{\mathbf{A}}=\bigvee_{n \in \mathbb{Z}} \operatorname{range}\left(A_{n}\right)$ by (26)). As mentioned earlier (Section 2), $\mathbf{A}$ is deterministic if and only if $V_{\mathbf{A}}:=\left.U_{\mathbf{A}}^{*}\right|_{\mathcal{K}_{0}^{\mathbf{A}}=\bigvee_{n \leq 0} \operatorname{range}\left(A_{n}\right)}$ is a unitary operator and $\mathbf{A}$ is purely non-deterministic (moving average of a white noise) if and only if $V_{\mathbf{A}}$ is a shift. More precisely the following corollary holds.

COROLlaRY 3.15. A is a deterministic (respectively a purely non-deterministic) stationary process if and only if $\operatorname{range}\left(A_{0}\right) \subset \mathcal{K}_{-1}^{\mathbf{A}}=\bigvee_{n \leq-1} \operatorname{range}\left(A_{n}\right)$ (respectively $\left.\bigcap_{n \leq 0} \bigvee_{m \leq n} \operatorname{range}\left(A_{m}\right)=\{0\}\right)$ and $\mathbf{A}$ satisfies one of the equivalent conditions (19), (20)+(21) or (24). 
Passing to the unilateral case, we provide necessary and sufficient conditions on a given sequence $\left\{A_{n}\right\}_{n \geq 0} \subset \mathcal{L}(\mathcal{H}, \mathcal{K})$ to assure a unitary solution to Problem $B$ :

Proposition 3.16. Let $\mathcal{K}_{0}=\bigvee_{n \geq 0} \operatorname{range}\left(A_{n}\right)$ and $\mathcal{K}_{1}=\bigvee_{n \geq 1} \operatorname{range}\left(A_{n}\right)$. Problem $B$ has a unitary solution if and only if

$$
\operatorname{dim}\left(\mathcal{K} \ominus \mathcal{K}_{0}\right)=\operatorname{dim}\left(\mathcal{K} \ominus \mathcal{K}_{1}\right)
$$

and $\left\{A_{n}\right\}_{n \geq 0}$ satisfies one of the equivalent conditions (15) or (16).

Proof. If Problem $B$ has a unitary solution $U$ on $\mathcal{K}$ then its restriction to $\mathcal{K}_{0}$ is clearly an isometric operator with range $\mathcal{K}_{1}$. Consequently $U\left(\mathcal{K} \ominus \mathcal{K}_{0}\right)=\mathcal{K} \ominus \mathcal{K}_{1}$, hence $\mathcal{K} \ominus \mathcal{K}_{0}$ and $\mathcal{K} \ominus \mathcal{K}_{1}$ have the same dimension.

Conversely, by (15) or (16), we obtain an isometric solution $V$ (on $\mathcal{K}$ ) for our problem. Then $U_{0}:=\left.V\right|_{\mathcal{K}_{0}}: \mathcal{K}_{0} \rightarrow \mathcal{K}_{1}$ is a unitary operator and the unitary solution we are looking for is given by the matrix representation

$$
U=\left(\begin{array}{cc}
U_{0} & 0 \\
0 & W
\end{array}\right)
$$

relative to orthogonal decompositions $\mathcal{K}=\mathcal{K}_{0} \oplus\left(\mathcal{K} \ominus \mathcal{K}_{0}\right)$ and $\mathcal{K}=\mathcal{K}_{1} \oplus\left(\mathcal{K} \ominus \mathcal{K}_{1}\right)$; $W$ is just an arbitrary unitary operator between $\mathcal{K} \ominus \mathcal{K}_{0}$ and $\mathcal{K} \ominus \mathcal{K}_{1}$.

Corollary 3.17. Let $\left\{h_{n}\right\}_{n \geq 0} \subset \mathcal{H}, \mathcal{K}_{0}=\bigvee_{n \geq 0} h_{n}$ and $\mathcal{K}_{1}=\bigvee_{n \geq 1} h_{n}$. Problem $A$ has a unitary solution if and only if

$$
\operatorname{dim}\left(\mathcal{K} \ominus \mathcal{K}_{0}\right)=\operatorname{dim}\left(\mathcal{K} \ominus \mathcal{K}_{1}\right)
$$

and $\left\{h_{n}\right\}_{n \geq 0}$ satisfies one of the equivalent conditions

$$
\left\langle h_{n+1}, h_{m+1}\right\rangle=\left\langle h_{n}, h_{m}\right\rangle, \quad m, n \geq 0
$$

or (17).

Remark 3.18. Define $U_{0}: \mathcal{K}_{0} \rightarrow \mathcal{K}_{1}$ by (11) (resp. by (10)). Any unitary solution of Problem $A$ (resp. Problem $B$ ) has the matrix representation given by (27). It is unique if and only if $\mathcal{K}=\mathcal{K}_{0}=\mathcal{K}_{1}$.

Remark 3.19. Define $V: \mathcal{K}_{0} \rightarrow \mathcal{K}_{0}$ by (11) (resp. by (10)) and suppose that it is isometric: Problem $A$ (resp. Problem $B$ ) has an isometric solution. The following situations can occur:

(a) $\operatorname{dim} \mathcal{K}_{0}<\infty$

In this case $V$ is unitary $\left(\mathcal{K}_{1}=\operatorname{range}(V)=\mathcal{K}_{0}\right)$ and can be obviously extended to a unitary solution on $\mathcal{K}$.

(b) $\operatorname{dim}\left(\mathcal{K} \ominus \mathcal{K}_{0}\right)<\infty$

Our problem has a unitary solution iff $\mathcal{K}_{0}=\mathcal{K}_{1}$ iff $V$ is unitary iff $h_{0} \in \mathcal{K}_{1}$ (resp. $\left.\operatorname{range}\left(A_{0}\right) \subset \mathcal{K}_{1}\right)$.

(c) $\operatorname{dim}\left(\mathcal{K} \ominus \mathcal{K}_{0}\right)=\infty$

- Since $\mathcal{K} \ominus \mathcal{K}_{1}=\left(\mathcal{K} \ominus \mathcal{K}_{0}\right) \oplus\left(\mathcal{K}_{0} \ominus \mathcal{K}_{1}\right)$, we deduce that the problem has a unitary solution $\left(\operatorname{dim}\left(\mathcal{K} \ominus \mathcal{K}_{0}\right)=\operatorname{dim}\left(\mathcal{K} \ominus \mathcal{K}_{1}\right)\right)$ if and only if $\operatorname{dim}(\mathcal{K} \ominus$ $\left.\mathcal{K}_{0}\right) \geq \operatorname{dim}\left(\mathcal{K}_{0} \ominus \mathcal{K}_{1}\right)$. In particular, the last condition holds true if $\operatorname{dim}(\mathcal{K} \ominus$ $\left.\mathcal{K}_{0}\right) \geq \operatorname{dim} \mathcal{K}_{0}$ (case considered in $\left.[8]\right)$. 
- If $\operatorname{dim} \mathcal{K}>\operatorname{dim} \mathcal{K}_{0}$ then $\operatorname{dim}\left(\mathcal{K} \ominus \mathcal{K}_{0}\right)=\operatorname{dim} \mathcal{K}=\operatorname{dim}\left(\mathcal{K} \ominus \mathcal{K}_{1}\right)$ and the problem has a unitary solution.

- If $0<\operatorname{dim}\left(\mathcal{K}_{0} \ominus \mathcal{K}_{1}\right)<\infty$ then our problem has a unitary solution if and only if $\operatorname{dim}\left(\mathcal{K} \ominus \mathcal{K}_{0}\right)=\infty$.

(d) $\operatorname{dim} \mathcal{K}_{0}=\aleph_{0}$

We distinguish two cases:

- $\operatorname{dim}\left(\mathcal{K} \ominus \mathcal{K}_{0}\right) \geq \aleph_{0}$ : the problem has a unitary solution (by (c));

- $\operatorname{dim}\left(\mathcal{K} \ominus \mathcal{K}_{0}\right)<\aleph_{0}$ : the problem has a unitary solution if and only if $\mathcal{K}_{0}=\mathcal{K}_{1}$ (by (b)).

3.4. Shift Solutions Lemma 3.20. (a) The restriction of a shift to one of its invariant (closed) subspaces is a shift;

(b) Any shift on a Hilbert space $\mathcal{H}_{0}$ can be extended to a shift on a given Hilbert space $\mathcal{H}$ containing $\mathcal{H}_{0}$ as a (closed) subspace.

Proof. (a) If $S$ is a shift on a Hilbert space $\mathcal{H}$ and $\mathcal{H}_{0}$ is any subspace of $\mathcal{H}$, invariant to $S$, then

$$
\bigcap_{n \geq 0} S^{n} \mathcal{H}_{0} \subset \bigcap_{n \geq 0} S^{n} \mathcal{H}=\{0\}
$$

(b) Suppose that $S_{0}$ is a shift on $\mathcal{H}_{0}$.

If $\mathcal{H}_{1}=\mathcal{H} \ominus \mathcal{H}_{0}$ is infinite dimensional then we can build a shift $S_{1}$ on $\mathcal{H}_{1}$ unitarily equivalent with the "standard shift" $T_{z} \otimes I$ on $H^{2}(\mathbb{T}) \otimes \mathcal{H}_{1}$ (the symbol " $\otimes$ " denotes here the hilbertian tensor product). This proves that $S=S_{0} \oplus S_{1}$ is a shift on $\mathcal{H}$ extending $S_{0}$.

If $\mathcal{H}_{1}$ is finite dimensional we proceed by induction. We only have to consider the case $\operatorname{dim} \mathcal{H}_{1}=1$. We take $h \in \mathcal{H}_{1}$ and $l \in \operatorname{ker} S_{0}^{*}$ with $\|h\|=\|l\|=1$. The operator $S$ defined on $\mathcal{H}=\mathcal{H}_{0} \oplus \mathcal{H}_{1}$ by

$$
S\left(h_{0}+\lambda h\right):=S_{0} h_{0}+\lambda l, \quad h_{0} \in \mathcal{H}_{0}, \lambda \in \mathbb{C}
$$

is linear and isometric. In addition, due to $S \mathcal{H} \subset \mathcal{H}_{0}$ we have

$$
\bigcap_{n \geq 0} S^{n} \mathcal{H}=\bigcap_{n \geq 1} S^{n-1}(S \mathcal{H}) \subset \bigcap_{n \geq 1} S_{0}^{n-1} \mathcal{H}_{0}=\bigcap_{n \geq 0} S_{0}^{n} \mathcal{H}_{0}=\{0\} .
$$

This shows that $S$ is a shift, as required.

The lemma above allows us to reduce the study of Problem $A$ (resp. Problem $B$ ) with shift solution to the case $\mathcal{K}=\bigvee_{n \geq 0} h_{n}\left(\operatorname{resp} . \mathcal{K}=\bigvee_{n \geq 0}\right.$ range $\left.\left(A_{n}\right)\right)$. More precisely the following proposition holds.

Proposition 3.21. Let $\left\{A_{n}\right\}_{n \geq 0} \subset \mathcal{L}(\mathcal{H}, \mathcal{K})$. Problem $B$ has a shift solution if and only if

$$
\bigcap_{n \geq 0} \bigvee_{m \geq n} \operatorname{range}\left(A_{m}\right)=\{0\}
$$

and $\left\{A_{n}\right\}_{n \geq 0}$ satisfies one of the equivalent conditions (15) or (16). 
Proof. Problem $B$ has a shift solution if and only if it has an isometric solution and the map (10) is a shift.

Corollary 3.22. Let $\left\{h_{n}\right\}_{n \geq 0} \subset \mathcal{H}$. Problem A has a shift solution if and only if

$$
\bigcap_{n \geq 0} \bigvee_{m \geq n} h_{m}=\{0\}
$$

and $\left\{h_{n}\right\}_{n \geq 0}$ satisfies one of the equivalent conditions (28) or (17).

REMARK 3.23. We can observe that, by Lemma 3.20, a stationary process $\mathbf{A}=$ $\left\{A_{n}\right\}_{n \in \mathbb{Z}}$ is a moving average of a white noise if and only if Problem $\mathrm{B}$ for $\left\{A_{-n}\right\}_{n \geq 0}$ has a shift solution (see also Corollary 3.15).

4. Some Applications. Let $\mathbf{T}=\left(T_{1}, T_{2}\right)$ be a commuting pair of contractions on a Hilbert space $\mathcal{H}$ and $D_{T_{1}}=\left(I-T_{1}^{*} T_{1}\right)^{1 / 2}, D_{T_{2}}=\left(I-T_{2}^{*} T_{2}\right)^{1 / 2}$ be the corresponding defect operators. Recall (from Section 2 ) that $\mathbf{T}$ has a regular unitary dilation if and only if

$$
\Delta_{\mathbf{T}}=I-T_{1}^{*} T_{1}-T_{2}^{*} T_{2}+T_{1}^{*} T_{2}^{*} T_{1} T_{2} \geq 0
$$

Equivalently, (29) can be rewritten as

$$
I-T_{1}^{*} T_{1} \geq T_{2}^{*}\left(I-T_{1}^{*} T_{1}\right) T_{2}
$$

or as

$$
\left\|D_{T_{1}} T_{2} h\right\| \leq\left\|D_{T_{1}} h\right\|, \quad h \in \mathcal{H} .
$$

We deduce that there exists a contraction $C$ on the defect space $\mathcal{D}_{T_{1}}=\overline{D_{T_{1}} \mathcal{H}}$ with

$$
C D_{T_{1}}=D_{T_{1}} T_{2}
$$

In other words, Problem $B$ for the sequence $\left\{D_{T_{1}} T_{2}^{n}\right\}_{n \geq 0}$ has a contractive solution.

By Theorem 3.1 (iii) we obtain that

$$
\sum_{m, m^{\prime}, n, n^{\prime}}\left\langle D_{T_{1}} T_{2}^{(m-n)^{+}+m^{\prime}}\left(h_{m, m^{\prime}}\right), D_{T_{1}} T_{2}^{(m-n)^{-}+n^{\prime}}\left(h_{n, n^{\prime}}\right)\right\rangle \geq 0,
$$

for every finite double sequence $\left\{h_{n, n^{\prime}}\right\}_{n, n^{\prime} \geq 0}$ of vectors in $\mathcal{H}$. Consequently, if we take $h_{n}=\sum_{n^{\prime}} T_{2}^{n^{\prime}}\left(h_{n, n^{\prime}}\right), n \geq 0$, our condition becomes

$$
\sum_{m, n}\left\langle T_{2}^{*(m-n)^{-}}\left(I-T_{1}^{*} T_{1}\right) T_{2}^{(m-n)^{+}} h_{m}, h_{n}\right\rangle \geq 0,
$$

for every finite sequence $\left\{h_{n}\right\}_{n \geq 0}$ of vectors in $\mathcal{H}$. We use the Naimark dilation theorem ([12], [13]; cf. Section 2) to obtain new positivity conditions for regularity.

THEOREM 4.1. The following conditions are equivalent:

(i) $\mathbf{T}$ has a regular unitary dilation; 
(ii) The function

$$
\mathbb{Z} \ni n \mapsto T_{2}^{* n^{-}}\left(I-T_{1}^{*} T_{1}\right) T_{2}^{n^{+}} \in \mathcal{L}(\mathcal{H})
$$

is $P D$ on $\mathbb{Z}$;

(iii) There exists a semispectral measure $F$ on the unit circle into $\mathcal{L}(\mathcal{H})$ such that

$$
\left(I-T_{1}^{*} T_{1}\right) T_{2}^{n}=\int_{\mathbb{T}} \bar{\lambda}^{n} d F(\lambda), \quad n \geq 0
$$

(iv) There exists a Hilbert space $\mathcal{K}, V \in \mathcal{L}(\mathcal{H}, \mathcal{K})$ and a unitary operator $U$ on $\mathcal{K}$ such that

$$
\left(I-T_{1}^{*} T_{1}\right) T_{2}^{n}=V^{*} U^{n} V, \quad n \geq 0 .
$$

(v)

$$
\sum_{m, n}\left\langle T_{1} T_{2}^{(m-n)^{+}} h_{m}, T_{1} T_{2}^{(m-n)^{-}} h_{n}\right\rangle \leq \sum_{m, n}\left\langle T_{2}^{(m-n)^{+}} h_{m}, T_{2}^{(m-n)^{-}} h_{n}\right\rangle,
$$

for every finite sequence $\left\{h_{n}\right\}_{n \geq 0}$ of vectors in $\mathcal{H}$.

The same kind of conditions hold if we intertwine $T_{1}$ with $T_{2}$.

We conclude the paper with some new characterizations for orthogonal projections.

Proposition 4.2. Let $P \in \mathcal{L}(\mathcal{H})$ with $P^{2}=P$ (idempotent). Then $P$ is an orthogonal projection if and only if

$$
\left\|P h+P^{*} h^{\prime}\right\|=\left\|P h^{\prime}+P^{*} h\right\|, \quad h, h^{\prime} \in \mathcal{H} .
$$

Proof. We consider the sequence $\left\{A_{n}\right\}_{n \geq 0}$ defined by

$$
A_{n}= \begin{cases}P, & \text { if } n \text { is even } \\ P^{*}, & \text { if } n \text { is odd }\end{cases}
$$

If there exists an isometry $V$ on $\mathcal{H}$ with $V P=P^{*}$ and $V P^{*}=P$ then

$$
P^{*} P=(V P) P=V P=P^{*}
$$

and $P$ is an orthogonal projection. Obviously (14) can be rewritten in the equivalent form (30).

Proposition 4.3. An operator $P \in \mathcal{L}(\mathcal{H})$ is an orthogonal projection if and only if

$$
\left\|P h+P h^{\prime}\right\| \leq\left\|h+P h^{\prime}\right\|, \quad h, h^{\prime} \in \mathcal{H} .
$$

Proof. We apply Theorem $3.1((i) \Leftrightarrow(i v))$ to the sequence $\left\{A_{n}\right\}_{n \geq 0}$ given by

$$
A_{0}=I \quad \text { and } \quad A_{n}=P(n \geq 1) .
$$

It is easy to observe that there exists a contraction $T$ with $T^{n}=P(n \geq 1)$ if and only if $P^{2}=P$ and $\|P \leq 1\|$ or, equivalently, $P$ is an orthogonal projection. By the other hand, (iv) take the form (31) with $h=h_{0}$ and $h^{\prime}=\sum_{n \geq 1} h_{n}$. 
Acknowledgements. We want to express our gratitude to Professor Jan Stochel for useful related discussions and comments. Our appreciation goes also to the referee for his remarks towards an improved final version of the paper.

\section{REFERENCES} $88-90$.

1. T. Ando, On a pair of commutative contractions, Acta Sci. Math. (Szeged) 24 (1963),

2. S. Brehmer, Über vertauschbare kontractionen des Hilberschen raumes, Acta Sci. Math. (Szeged) 22 (1961), 106-111.

3. C. Foiaş and A. E. Frazho, The Commutant Lifting Approach to Interpolation Problems, Operator Theory: Advances and Applications 44, Birkhäuser-Verlag, Basel, 1990.

4. R. K. Getoor, The shift operator for non-stationary stochastic processes, Duke Math. J. 23 (1956), 175-187.

5. P. R. Halmos, Shifts on Hilbert spaces, J. Reine Angew. Math. 208 (1961), 102-

6. H. Helson and D. Lowdenslager, Prediction theory and Fourier series in several variables I, Acta Math. 99 (1958), 165-202.

7. H. Helson and D. Lowdenslager, Prediction theory and Fourier series in several variables II, Acta Math. 106 (1961), 175-213.

8. Z. J. Jabłoński and J. Stochel, Subnormality and operator multidimensional moment problems, J. London Math. Soc. (2) 71 (2005), 438-466.

9. Y. Kakihara, Multidimensional Second Order Stochastic Processes, Series on Multivariate Analysis 2, World Scientific Publ. Co., Inc., River Edge, NJ, 1997.

10. P. Masani, Orthogonally scattered measures, Adv. in Math. 2 (1968), 61-117.

11. P. Masani, Quasi-isometric measures and their applications, Bull. Amer. Math. Soc. 76 (1970), 427-528.

12. M. A. Naimark, On a representation of additive operator set functions, C.R. (Doklady) Acad. Sci. URSS 41 (1943), 359-361.

13. M. A. Naimark, Positive definite operator functions on a commutative group, Bull. Acad. Sci. URSS 7 (1943), 237-244.

14. A. Olofsson, Operator valued $n$-harmonic measure in the polydisc, Studia Math. 163 (2004), 203-216.

15. D. Popovici, On $C_{0}$. multi-contractions having regular dilation, Studia Math. 170 (2005), 297-302.

16. D. Popovici, Regular dilations on Kreĭn spaces, preprint (2004).

17. D. Popovici and Z. Sebestyén, Sebestyén moment problem: The multidimensional case, Proc. Amer. Math. Soc. 132 (2004), 1029-1035.

18. Z. Sebestyén, Moment theorems for operators on Hilbert space, Acta Sci. Math. (Szeged) 44 (1982), 165-171.

19. Z. Sebestyén, Moment theorems for operators on Hilbert space II, Acta Sci. Math. (Szeged) 47 (1984), 101-106.

20. Z. Sebestyén, A moment theorem for contractions on Hilbert spaces, Acta Math. Hung. 47 (1986), 391-393.

21. Z. Sebestyén, Moment problem for dilatable semigroups of operators, Acta Sci. Math. 45 (1983), 365-376.

22. Z. Sebestyén, Moment type theorems for $*$-semigroups of operators on Hilbert space I, Ann. Univ. Sci. Budapest, Eötvös Sect. Math. 26 (1983), 213-218.

23. I. Suciu and I. Valuşescu, Factorization theorems and prediction theory, Rev. Roum. Math. Pures et Appl. 23 (1978), 1393-1423.

24. I. Suciu and I. Valuşescu, A linear filtering problem in complete correlated actions, J. Multivariate Analysis 9 (1979), 599-613.

25. B. Sz.-Nagy, Bemerkungen zur vorstehenden Arbeit des Herrn S. Brehmer, Acta Sci. Math. (Szeged) 22 (1961), 112-114. 

$87-92$.

26. B. Sz.-Nagy, Sur les contractions de l'espace de Hilbert, Acta Sci. Math. 15 (1953),

27. B. Sz.-Nagy and C. Foiaş, Harmonic Analysis of Operators on Hilbert Space (Akadémiai Kiadó, Budapest, North-Holland, Amsterdam, 1970).

28. N. Wiener and P. Masani, The prediction theory of multivariate stochastic processes I, Acta Math. 98 (1957), 111-150.

29. N. Wiener and P. Masani, The prediction theory of multivariate stochastic processes II, Acta Math. 99 (1958), 93-139. 